\title{
L'eau : inodore, incolore et insipide ? Un mensonge phraséologiquement inacceptable
}

\section{Díaz Rodríguez, Cristian}

Université de Strasbourg (LiLPa) - Universidad de La Laguna, cdiazrodriguez@unistra.fr

\section{Resumen}

El agua se presenta como un elemento altamente productivo para la formación de unidades fraseológicas. La no composicionalidad inherente a este tipo de unidades pone en cuestión la tríada definitoria : incolora, inodora e insípida. Un análisis detallado de las combinaciones eaux / marée + AdjChr, que tenga en cuenta la polisemia de los términos acuosos, nos permitirá organizar de forma más eficiente el cajón de sastre lexicográfico recogido bajo la etiqueta « combinaciones frecuentes ».

Palabras clave: fraseología; colocaciones; locuciones; compuestos colocacionales; compuestos locucionales.

\section{Résumé}

L'eau s'avère être un élément hautement productif pour la création d'unités phraséologiques. La noncompositionnalité inhérente à ce type d'unités met en question la triade définitoire : incolore, inodore, insipide. Une analyse détaillée des combinaisons ayant la forme eaux / marée + AdjChr et qui reflètent la polysémie des termes aqueux nous permettra d'organiser de manière plus efficiente le fourre-tout lexicographique des « combinaisons fréquentes ».

Mots-clés : phraséologie ; collocations ; locutions ; composés collocationnels ; composés locutionnels.

\begin{abstract}
The water is a highly productive element to create phraseological units. The defining triad : colourless, odourless, insipid is called in question by these units' inherent non compositionality. A deep analyse of the structures eaux / marée + AdjChr reflecting the aqueous' polysemy will let us organise in a more efficient way the lexicographic jumble of « frequent combinations ».
\end{abstract}

Keywords : phraseology ; collocations ; idioms ; collocational compounds ; idiomatic compounds. 
Où vas-tu, insensé que tu es ? Tu vas porter de l'eau à la rivière.

\section{Introduction}

Nous avons appris à l'école que l'eau était un liquide inodore, incolore et insipide. Nous l'avons accepté parce que l'expérience empirique le confirmait et surtout parce que c'était le « prof » qui le disait. Cependant, cette affirmation, répétée sous forme de mantra, n’était qu’un infâme mensonge, clair comme l'eau de roche, du moins sous une perspective phraseólogique.

L'eau douce, l'eau salée ou l'eau-de-vie ont bel et bien un goût particulier. De même, l'eau de parfum, l'eau de toilette ou l'eau de Cologne sont réputées pour leur fragrance. Quant à la couleur, le spectre chromatique teint les eaux de syntagmes qui vont des noms propres, e. g. la mer Noire, le fleuve Jaune, aux unités phraséologiques (=UP) dénominatives, e. g. le grand bleu - l’Atlantique -, la grande bleue - la Méditerranée.

Dans cette communication, nous nous focaliserons sur l'étude des UP contenant simultanément une lexie chromatique et les substantifs eau(x) / marée, e. g. eaux noires, eaux grises, marée noire, marée rouge. Pour ce faire, nous extrairons toutes les cooccurrences présentes dans quatre dictionnaires monolingues possédant un moteur de recherche : Le Petit Robert, Le Trésor de la Langue française informatisé, Le Littré et Le Larousse.

Après avoir proposé une brève définition de ce que nous entendons par UP dans cette étude (§1), nous réfléchirons aux particularités de ces UP, situées entre les collocations, les locutions nominales et les noms composés. Elles servent à nommer un hyponyme de l'espèce indiquée par la base, mais, en même temps, détruisent l'un des sèmes inhérents à celleci : l' « incolorité » (§2). Nous finirons cette présentation par l'étude de cas dans lesquels convergent en une même UP un sens phraséologique traditionnel et un néologisme à haute idiomaticité (§3) - calque de l'espagnol -, e. g. marea verde (éducation), marea granate (« expatriés ») vs marée verte, marée grenat.

\section{L’unité phraséologique : une définition turbulente}

Selon l'approche fonctionnelle ${ }^{1}$ de la phraséologie, à laquelle nous adhérons, une UP est une combinaison, d'au moins deux lexies, caractérisée par un certain degré de figement.

En termes structurels, le figement implique :

a. le blocage des propriétés transformationnelles :

(1) Il n'a pas tant de choses à faire, mais il se noie dans un verre d'eau

(2) *C'est dans l'eau de ce verre qu'il se noie

(3) *L'énorme quantité de travail l'a noyé dans un verre d'eau

b. l'inaltérabilité de l’ordre des constituants :

$$
\text { Le Sporting Strasbourg a sué sang et eau pour remporter cette victoire }
$$

*Le Sporting Strasbourg a sué eau et sang pour remporter cette victoire

c. la restriction paradigmatique des constituants :

$$
\text { Chat échaudé craint l'eau froide }
$$

$$
\text { *Félin ébouillanté a peur de l'eau presque gelée }
$$

${ }^{1}$ Pour l'approche statistique de la phraséologie, la simple haute fréquence de cooccurrence sert à définir les UP. 
Néanmoins, les UP peuvent éventuellement manifester une certaine souplesse qui se traduit par des modifications au niveau morphosyntaxique - diminutifs, augmentatifs, intensificateurs, atténuateurs, troncations, ellipses, etc. - pouvant devenir ensuite des variations également conventionnalisées :

$$
\begin{aligned}
& \text { Il n'a pas inventé l'eau tiède / l'eau chaude } \\
& \text { Tant va la cruche à l'eau... (qu'à la fin elle se casse / se brise) } \\
& \text { Au début, il était très exigeant, mais après il a mis (un peu d') de l'eau dans son vin }
\end{aligned}
$$

Quant au contenu, le sens phraséologique s’inscrit dans le langage figuré conventionnalisé (Dobrovol’skij et Piirainen, 2005) et, par conséquent, il s’agit d’un sens idiomatique ${ }^{2}$ dérivé de la réinterprétation du matériel linguistique préexistant $^{3}$ :

Avec plus de 20\% de la population mondiale, mais seulement $7 \%$ des ressources en eau, la Chine a soif d'or bleu $\rightarrow$ 'or bleu'= eau $\neq$ ₹ 'or' + 'bleu'

Cela n'implique pas que le sens de chaque composante subisse une réinterprétation sémantique individuelle. De fait, maintes UP n’ont subi qu'une réinterprétation partielle, e. g. vivre d'amour et d'eau fraîche $\rightarrow$ '[vivre] de façon insouciante' ; voire aucune réinterprétation se ressembler comme deux gouttes d'eau.

Cependant, le caractère conventionnalisé opérant lors du processus de phraséologisation octroie toujours un enrichissement à l'ensemble qui garantit la survie de l'UP face à d'autres lexies simples quasi-synonymiques. Si nous reprenons l'exemple d'or bleu, nous pouvons constater qu'il représente une manière alternative - dénomination secondaire - pour dénoter l'eau, puisqu’il inclut, de surcroît, l'enrichissement « précieux parce qu'épuisable » :

OR BLEU $=$ EAU + \{ENRICHISSEMENT $\}$

ENRICHISSEMENT = «précieux parce qu’épuisable »

OR BLEU = EAU つ « précieux parce qu'est épuisable »

Fig. 1. Sens phraséologique enrichi de l’UP or bleu

L’enrichissement ne saurait pourtant se limiter au plan sémantique. Certaines UP présentent des enrichissements qui contraignent leur utilisation dans le discours vis-à-vis du registre, de l'implication du locuteur, des buts discursifs poursuivis ou encore des contraintes situationnelles (enrichissement pragmatique) :

$$
\text { Compte là-dessus et bois de l'eau! }
$$

À l'instar de L. Timofeeva (2012), nous considérons que le sens phraséologique est enrichi par l’incorporation

\footnotetext{
2 À l'instar de J.-C. Anscombre, nous entendons l’idiomaticité comme « une relation conventionnelle entre deux ou plus de deux éléments de la langue [...]. Cette idiomaticité provient de traits culturels qui ont fini par se lexicaliser. » (2011: 34).

${ }^{3}$ D’après Baranov et Dobrovols’skij (1996 : 52, apud Timofeeva, 2012) moyennant la réinterprétation $R$, le sens 'A' d'une expression A devient le sens 'B'.

${ }^{4}$ Créé sur le modèle d’or noir signifiant 'pétrole’, l’or bleu peut également faire référence au gaz en tant que ressource épuisable.
} 
d'inférences à différents niveaux de conventionnalisation. Ainsi, par exemple, même si se brosser les dents et se brosser les cheveux sont des syntagmes sémantiquement compositionnels, ils sont bel et bien des UP parce qu'ils déclenchent certaines inférences par défaut :

Tableau 1. Sens phraséologique enrichi des UP se brosser les cheveux et se brosser les dents

\begin{tabular}{|c|c|c|}
\hline & Compositionnalité sémantique & Inférence \\
\hline Se brosser les cheveux & [se $]+[$ brosser $]+[$ les cheveux $]$ & $\begin{array}{l}\text { + Avec une brosse à cheveux } \\
\text { + À sec (la présence de l'eau n'est pas exigée) } \\
\text { + Aucun produit supplémentaire n'est exigé }\end{array}$ \\
\hline Se brosser les dents & [se] + [brosser] + [les dents] & $\begin{array}{l}\text { + Avec une brosse à dents } \\
\text { + La présence de l'eau est exigée } \\
\text { + L’utilisation de dentifrice est présumée }\end{array}$ \\
\hline
\end{tabular}

L’eau, en tant qu'élément intégrant la réalité référentielle quotidienne des locuteurs, apparaît comme un terme enclin aux exercices de réinterprétation sur lesquels est fondée la phraséologisation de syntagmes. Aussi l'eau apparaît-elle comme élément composant de nombre d’exemples intégrant le continuum phraséologique :

\section{Tableau 2. UP contenant la lexie eau(x)}

\begin{tabular}{|c|c|}
\hline Parémies & $\begin{array}{l}\text { Il n'est pire eau que l'eau qui dort, tant va la cruche à l'eau qu'à la fin elle se casse, chat échaudé } \\
\text { craint l'eau froide }\end{array}$ \\
\hline & $\begin{array}{l}\text { [être] comme l'eau et le feu, se noyer dans un verre d'eau, une tempête dans un verre d'eau, mettre de } \\
\text { l'eau dans son vin, faire de l'eau, faire eau, [être] la goutte d'eau qui fait déborder le vase, [être] une } \\
\text { goutte d'eau dans l'océan / la mer, passer de l'eau sous les ponts, apporter de l'eau au moulin, coup }\end{array}$ \\
\hline Locutions & $\begin{array}{l}\text { d'épée dans l'eau, ne pas avoir inventé l'eau tiède / chaude, être / rester le bec dans l'eau, jeter le bébé } \\
\text { avec l'eau du bain, s'en aller en eau de boudin, vivre d'amour et d'eau fraîche, au fil de l'eau, pêcher } \\
\text { en eau trouble, [être] comme poisson dans l'eau, tomber / être à l'eau, se jeter à l'eau, avoir la tête } \\
\text { sous l'eau, aller à vau-l'eau, avoir de l'eau dans le gaz, à l'eau de rose }\end{array}$ \\
\hline Collocations & $\begin{array}{l}\text { prendre de l'eau, se ressembler comme deux gouttes d'eau, [marcher] au bord de l'eau, nager sous } \\
\text { l'eau, jeter qqn à l'eau, flotter dans l'eau, mettre [un navire] à l'eau, roman à l'eau de rose, l'eau + } \\
\text { \{jaillir de, couler, courir, s'écouler, pénétrer dans, s'infiltrer dans, déborder, ruisseler, tourbillonner, } \\
\text { bouillir, bouillonner, geler, se condenser, se faire rare, s'évaporer\}, s'imbiber d'eau, économiser de } \\
\text { l'eau, gaspiller de l'eau, couper l'eau, etc. }\end{array}$ \\
\hline Pragmatèmes & Compte là-dessus et bois de l'eau! \\
\hline Noms composés & $\begin{array}{l}\text { bouteille d'eau, cours d'eau, chute d'eau, flaque d'eau, couleuvre d'eau, araignée d'eau, lentille d'eau, } \\
\text { goutte d'eau, eau-de-vie, eau-forte, eaux-vannes }{ }^{5} \text {, eau de Seltz, eau de Javel, eau oxygénée, eau régale, } \\
\text { eaux-mères, eau de Cologne, eau de parfum, eau de toilette, eau de rose, eau de lavande, eau de fleurs, } \\
\text { eau de pluie, eau du robinet, eau de mer, eau de surface, eau de roche, eaux de ruissellement, eau de } \\
\text { fleuve, eau de la fontaine, eau du lac, eau du ruisseau, eau de source }\end{array}$ \\
\hline
\end{tabular}

Cependant, il y a tout un groupe de combinaisons qui sillonnent l'immensité de l'océan phraséologique, qui nagent entre au moins deux eaux, d'ailleurs très turbulentes. Nous parlons des combinaisons qui prennent la forme eau $+A d j$, dans lesquelles l'adjectif apporte des informations concernant, entre autres, sa température - eau chaude, eau glacée -, sa couleur - eau bleue, eau jaune -, sa limpidité - eau boueuse, eau limpide -, son mouvement - eau calme, eau rapide -,

\footnotetext{
${ }^{5}$ Nous sommes conscients que la nature phraséologique des composés suscite le débat, surtout si nous avons affaire à des syntagmes synthétiques, e. g. presqu'île, eauxvannes, hydrophobe. À notre avis, seul les composés complètement soudés, e. g. hydrophobe, sont exclus de la phraséologie, car ils sont déphraséologisés (García-Page, 2012), même s'ils ont subi une réinterprétation : Jean ne s'approche pas de la piscine, on dirait un hydrophobe vs La cire est un matériel hydrophobe, c'est-à-dire elle n'est pas dissoluble dans l'eau.
} 
sa saveur - eau salée, eau amère -, ou encore sa provenance - eaux fluviales, eaux souterraines - :

Eau(x) \{thermale, baptismale, bénite, lustrale, sacrée, sanctifiée, salée, courante, gazéifiée, minérale, pétillante, plate, bouillante, bouillie, brûlante, bouillonnante, chaude, tiède, fraîche, froide, glacée, calcaire, dure, contaminée, empoisonnée, impropre à la consommation, non potable, polluée, insipide, claire, propre, pure, purifiée, buvable, potable, propre à la consommation, salubre, cristalline, diaphane, limpide, translucide, transparente, douce, marine, océanique, vive, profonde, souterraine, superficielle, sombre, assoupie, calme, dormante, immobile, lisse, paisible, stagnante, tranquille, agitée, capricieuse, dangereuse, déchaînée, houleuse, tourmentée, tumultueuse, turbulente, boueuse, bourbeuse, limoneuse, sableuse, ferrugineuse, soufrée, sulfureuse, laiteuse, trouble, sale, souillée, gluante, grasse, huileuse, saumâtre, croupie, distillée, gazeuse, qui pique, plate, lourde, littorales, territoriales, fluviales, usagées, usées, de ruissellement, pluviales, résiduaires\}

La totalité des dictionnaires consultés incluent un important répertoire de ces combinaisons sans qu’il existe pourtant un critère servant à distinguer le type d'UP auquel nous avons affaire. De fait, en se retranchant derrière l'imprécision du terme «combinaisons fréquentes », les ouvrages lexicographiques font l'amalgame des locutions, collocations, noms composés et même de combinaisons qui font plutôt partie de la syntaxe libre (cf. section SYNT du TLFi). Certes, pour la plupart des cas, la composante eau a subi une réinterprétation qui entraine le non-respect d'au moins un des traits définitoires, ce qui les situe dans la sphère phraséologique, mais il est évident qu'il faut établir un filtrage à l'intérieur. Dans ce travail, nous n'étudierons que les syntagmes ayant la forme : eau + AdjChr (=adjectif chromatique).

\section{Des eaux bigarrées : une exception qui devient la norme}

L'eau est liée à la couleur, au moins d'un point de vue conventionnel. Comme le signale M. Pastoureau (1992), grâce aux couleurs situées sur les robinets, tout le monde peut identifier sans trop de peine dans quel sens il faut tourner la poignée du robinet afin de calibrer la température de l'eau : bleu - eau froide, rouge - eau chaude. En Alsace, comme d'ailleurs dans d'autres régions, la couleur rouge des bouteilles d'eau n'a rien à voir avec la température mais avec le type d'eau. Aussi une Carola rouge contiendra-t-elle de l'eau pétillante, alors que si l'on veut de l'eau plate, il faudra acheter une bouteille de Carola bleue, la verte étant réservée à l'eau finement pétillante ${ }^{6}$. Les couleurs des drapeaux qui président les côtes constituent un autre exemple des relations conventionnelles existant entre les couleurs et l'eau, en l'occurrence l'état de la mer : rouge - baignade interdite, orange - baignade dangereuse mais surveillée, vert - baignade surveillée et absence de danger, bleu - limite de zone surveillée. Cependant, dans tous ces exemples, la relation eau-couleur n'est que tangentielle puisque c'est toujours un référent autre que le liquide lui-même qui est caractérisé par le sème chromatique.

Au plan linguistique, l'eau peut être qualifiée moyennant une couleur ${ }^{7}$ et ce, malgré son trait définitoire d'incolorité. Dans le cadre de la syntaxe libre, cela se produit si la coloration de l'eau reflète une réalité ontologique produite soit par l'action d'une couleur-pigment ou d'une teinture en contact avec le liquide aqueux (13-15), soit par une perception d'une couleur lumière (16-18) :

L'eau de l'étang est de plus en plus verte

Il faudrait changer l'eau du seau, elle est trop noire

L'eau qui sortait de la machine à laver était rouge, je crains que mes chemises blanches ne soient teintes

(16) J'aimerais bien être à Cuba avec ses plages de sable blanc et ses eaux turquoise

Le bateau s'est noyé dans les eaux bleues de l'océan profond

Les eaux noires des fleuves du bassin amazonien, dont le Rio Negro, sont acides et contiennent moins

\footnotetext{
${ }^{6}$ Cf. Eichenlaub (2011).

${ }^{7}$ Cette relation est bidirectionnelle, car certains noms de couleurs sont également construits à partir de référents hydriques, e. g. bleu marine, vert d'eau, le bleu / vert aiguemarine (même si l'aigue-marine couleur fait référence à un minéral, ce dernier prend son nom de la couleur des eaux turquoise pâles).
} 
L'eau : inodore, incolore et insipide ? Un mensonge phraséologiquement inacceptable

de nutritifs

Cette coloration empirique ${ }^{8}$ est à la base de maintes hydronymes et cela indépendamment du type de dénomination qu'ils reçoivent : primaire - mer Blanche, mer Noire, mer Jaune, mer Rouge, le fleuve Jaune, le fleuve Bleu, le fleuve Río Negro, le lac Noir, le lac Blanc, le lac Vert, le lac Jaune, le lac Violet - ou secondaire - la grande bleue (la Méditerranée), le grand bleu (l'Atlantique).

A priori, nous pourrions penser que le non-respect de ce trait définitoire pourrait bien être la conséquence d'un processus de réinterprétation subi par la lexie eau. Néanmoins, nous avons affaire ici à un terme polysémique, car la triade incolore, inodore et insipide ne définit que l'eau pure et distillée. Autrement dit, ces caractéristiques ne sont pas définitoires du terme super-ordonné eau, mais d'un type particulier et hyponymique qui, de par son importance, est parvenu à s'imposer comme prototype. Ce n’est pas sans raison que cette définition est présentée comme première acception dans tous les dictionnaires consultés :

Liquide incolore, inodore, transparent et insipide lorsqu'il est pur $\left(\mathrm{H}_{2} \mathrm{O}\right)$ (Petit Robert)

Liquide incolore, inodore et sans saveur à l'état pur, formé par combinaison d'hydrogène et d'oxygène, de formule chimique $\mathrm{H}_{2} \mathrm{O}$; un des quatre éléments de la physique ancienne. (TLFi)

Corps liquide à la température et à la pression ordinaires, incolore, inodore, insipide, dont les molécules sont composées d'un atome d'oxygène et de deux atomes d'hydrogène. (Larousse)

Substance liquide, transparente, sans saveur ni odeur, réfractant la lumière et susceptible de dissoudre un grand nombre de corps. (Littré)

Même si cette nouvelle définition d'eau nous éloigne de la réinterprétation et, par conséquent, du domaine phraséologique, l'AdjChr qui co-apparaît nous y reconduit puisque, dans tous les cas, il va apporter des informations autres que la couleur. En d’autres termes, l’AdjChr a été réinterprété.

Tableau 3. Signifiés des UP ayant la structure eau(x) + AdjChr

\begin{tabular}{|c|c|}
\hline Eau blanche & $\begin{array}{l}\text { 1. Liqueur blanchâtre et astringent formé d'un mélange d'eau et d'extrait de Saturne (sous-acétate } \\
\text { de plomb liquide). } \\
\text { 2. [Eau] dans laquelle on a jeté de la farine ou du son pour la faire boire aux animaux } \\
\text { domestiques, notamment aux chevaux. }\end{array}$ \\
\hline Eau rougie & [Eau] mêlée d’une légère quantité de vin rouge \\
\hline Eau jaune & Pastis préparé \\
\hline Eau céleste & $\begin{array}{l}\text { Liquide bleu obtenu en versant de l’ammoniaque liquide dans de l'eau distillée tenant en } \\
\text { dissolution du sulfate de cuivre. }\end{array}$ \\
\hline Eaux noires & $\begin{array}{l}\text { [Eaux] usées provenant des toilettes et devant subir des traitements plus intensifs que les eaux } \\
\text { grises }\end{array}$ \\
\hline Eaux grises & [Eaux] résiduaires savonneuses, provenant de douches, eau de vaisselle et lessive \\
\hline Eaux blanches & $\begin{array}{l}\text { 1. [Eaux] polluées, notamment dans l'industrie des papeteries. } \\
\text { 2. [Eaux] de lavage des laiteries et des salles de traite, contenant les différents détergents utilisés } \\
\text { ainsi que des résidus de lait }\end{array}$ \\
\hline
\end{tabular}

\footnotetext{
${ }^{8}$ Il se peut que les AdjChr associés aux eaux ne correspondent pas exactement à des notions chromatiques, mais à d'autres acceptions liées à ces couleurs. Ainsi, par exemple, l’AdjChr noir pourrait faire référence à des épisodes funestes ayant eu lieu dans ces eaux, e. g. le fleuve Mavroneri (Eaux Noires) qui, malgré sa clarté et sa pureté, est censé être le fleuve Styx et peut, par conséquent, mener à la la mort si l'on en boit. Autrement dit, au lieu de manifester le sème chromatique, l'adjectif noir signifiera plutôt « dangereux ».
} 
Le problème surgit au moment du filtrage de ces eaux visant à les classer dans le compartiment phraséologique correspondant. Trois cas de figure sont à distinguer :

- Locution nominale : Les composantes de l’UP, après la réinterprétation, adoptent un sens unitaire. Par conséquent, la réinterprétation partielle du syntagme n'est pas possible. C'est pourquoi, tous les syntagmes dont le signifié équivaut à eau + ' $\mathrm{X}$ ', e. g. eaux noires : eaux $+[\mathrm{X}=$ 'usées provenant des toilettes'] seront exclus. En revanche, une combinaison telle que marée noire, entendue comme 'phénomène regrettable s'étendant inexorablement' est bel et bien une locution nominale ( $c f$. §3).

\section{Élections en France : marée noire, le FN en tête dans six régions}

- Collocation : Le sens global de la combinaison n'est pas unitaire, mais compositionnel, i. e. 'base' + 'collocatif'. Éventuellement, le sens du collocatif peut être le résultat d’une réinterprétation, mais il doit être entendu comme un cas de polysémie conditionnée par la présence de la base. Les composantes de l'UP entretiennent une relation argument-prédicat (Tutin, 2013). Cela implique que la combinaison ne pourra pas servir à nommer un hyponyme de la base mais à la spécifier ou à la qualifier. En l’occurrence, l’AdjChr apporte un sens spécifique qui peut être expliqué par une fonction lexicale non standard ( $c f$. Mel'čuk, 2013), e. g. eau rougie = 'eau' + 'mêlée d'une légère quantité de vin rouge' ; eau blanche = 'eau' + 'dans laquelle on a jeté de la farine ou du son'.

- Nom composé: Les noms composés servent à désigner une réalité référentielle concrète en établissant une délimitation qui la transforme en une classe distinguable vis-à-vis des autres. Par conséquent, les noms composés dénomment, identifient et classent en même temps. Comme dans le cas des syntagmes, nous pouvons distinguer deux types de noms composés syntagmatiques phraséologiques :

- Composé locutionnel : Les composantes de ces noms composés ont été réinterprétées globalement et le résultat est un sens unitaire, ce qui les assimile aux locutions. Nous considérons, à l'instar de M. GarcíaPage, que la frontière moins instable entre locutions et noms composés se trouve au plan sémantique :

Les composés dénotent des classes dans la mesure où ils permettent de classifier les entités qu'ils nomment alors que les locutions prêtent des propriétés aux référents auxquels elles s’appliquent [...] ; [la] fonction primordiale [d’une locution] est de dénoter des qualités ou des propriétés pouvant s’appliquer à un nombre de réalités vastes et hétérogènes [...] (2012:102).

Nous pourrions considérer comme des composés locutionnels les combinaisons eau blanche, eau céleste et eau jaune, appellations populaires (dénominations secondaires) surgies face à des termes plus techniques. Même si la composante eau fait partie de la composition du produit, elle ne saurait pas s'y réduire. Linguistiquement, cela équivaudrait à dire que l'eau est intégrée dans les combinaisons, non pas comme élément compositionnel (le sens reste toujours unitaire) mais comme un sème.

\section{Je bois du pastis pour protéger la couche d'eau jaune}

- Composé collocationnel : Du fait de leur statut de noms composés, ce type sert également à dénoter une classe d'entités. Cependant, il ne se produit pas une réinterprétation globale des composantes. Au contraire, la base ${ }^{9}$ du composé collocationnel, sera toujours compositionnelle et servira à indiquer l'hypéronyme dont le composé indique l'hyponyme, e. g. eau douce est une classe d'eau. Comme nous pouvons le constater, si la relation établie entre la base et le composé suit la forme argument-prédicat, nous parlerons de collocation. $\mathrm{Si}$, a contrario, la relation est hypéronyme - trait distinguant un hyponyme, nous parlerons de composé collocationnel.

La distinction locution nominale vs composé locutionnel est plutôt satisfaisante, en revanche, la frontière entre collocation et composé collocationnel s'avère plutôt floue. De fait, la résolution de ce dilemme se réduit à la déjà traditionnelle

\footnotetext{
${ }^{9}$ Par similitude avec les collocations syntagmatiques, nous appellerons base (nom tête) et collocatif les deux termes faisant partie du nom composé.
} 
dichotomie adjectifs qualificatifs vs adjectifs classifieurs (cf. Girardin, 2005), de sorte que le collocatif des collocations fonctionnerait comme un adjectif qualificatif alors que celui des composés collocationnels fonctionnerait comme un adjectif classifieur.

Parmi les adjectifs non prédicatifs, certains établissent une classification du nom-tête tout en inscrivant celui-ci dans un paradigme, tel est le cas de vin rouge, vin blanc, vin rosé, pour reprendre l'exemple le plus rebattu. Ces adjectifs cumulent des propriétés classifiantes et paradigmatisantes, tout en s’individualisant des unités qui sont traditionnellement ainsi dénommées (Girardin, 2005 : 59).

Gross définit les adjectifs classifieurs comme ceux qui « permettent de désigner un élément dans une série » (2001 apud Girardin, 2005 : 61). De son côté, Ch. Girardin indique que l'adjectif classifieur est souvent un adjectif primaire qui, en outre, ne constitue pas une caractéristique inhérente du mot tête, mais qui pourtant l'inscrit dans une sous-classification. Nous pouvons constater que les AdjChr, sous certaines circonstances, peuvent remplir ces conditions, e. g. vin blanc, vin rouge, vin rosé.

Cependant, l’AdjChr qui accompagne la lexie eaux - eaux blanches, eaux noires, eaux grises - est dépourvu de sème chromatique $^{10}$ et, par conséquent, ces caractéristiques doivent être nuancées. Comment devons-nous classer l’AdjChr noir dans eaux noires et marée noire ? S'agit-il d'une classe d'eau ou simplement d'une qualification de l'eau ? Cette délimitation n'est pas simple, d'autant plus qu'en dehors de la combinaison, les adjectifs classifieurs peuvent fonctionner comme des adjectifs prédicatifs. Ainsi, S. Whittaker (2002 : 99) note que « La plupart des adjectifs ont un statut mixte : ils sont tantôt classifiants, tantôt qualifiants ».

On m'a dit d'apporter du vin blanc et j'ai acheté un pinot gris d'Alsace.

J'ai vu sa nouvelle voiture blanche

Ch. Girardin, de son côté, préconise l'utilisation d'une série de critères afin de identifier un adjectif classifieur. Nous les comparerons à chaque fois avec la collocation café noir :

a. postposition - spécifie une espèce à l’intérieur d’un genre :

Les eaux noires / *noires eaux ne sont pas utilisées pour arroser

Je n'ai pris qu'un café noir / *noir café

b. impossibilité de fonctionnement comme attribut sans qu'il y ait de changement de sens :

\#Les eaux d'arrosage ne sont pas noires $\rightarrow$ Les eaux d'arrosage sont des eaux noires \#Le café que je bois est noir $\rightarrow$ *Le café que je bois, c'est du café noir

c. blocage de toute graduabilité :

$$
\begin{aligned}
& \text { \#Les eaux qu'on réutilise sont très noires } \\
& \text { \#Le café que je bois est très noir }
\end{aligned}
$$

d. effacement fréquent du nom-tête propre du domaine de la terminologie :

Pour l'arrosage, à Strasbourg on réutilise les eaux noires et à Schiltigheim les grises

* Je vais prendre un café au lait et mon collègue un noir

Eu égard aux exemples, nous pouvons conclure que les différences apparues entre collocations et composés collocationnels sont liées au fonctionnement du collocatif. Dans le cas des collocations, il prédique une qualité particulière de la base - extensible éventuellement aux hyponymes subordonnés - sans établir des sous-classes à l’intérieur.

\footnotetext{
${ }^{10}$ Le même cas est observable dans les UP vin gris, vin bleu, vin vert.
} 

Tous les matins, je bois un café [décaféiné, arabica, robusta, Kopi Luwak, *noir] hyponymes de café Tous les matins, je bois un café [décaféiné, arabica, robusta, Kopi Luwak] hyponymes de café noir

Le collocatifs des noms composés collocationnels, en revanche, ne prédiquent pas de caractéristiques d'une base générale, mais ils sélectionnent l'une des sous-classes paradigmatiques de la série dont la base est l'hypéronyme.

Dans cette centrale, on réutilise les eaux [salées, douces, non potables, noires, blanches, grises] hyponymes d'eaux

Dans cette centrale, on réutilise les eaux [salées, douces, non potables] hyponymes d'eaux *noires / *grises / *blanches

Aussi pouvons-nous affirmer que les UP eaux noires, eaux grises, eaux blanches constituent une série paradigmatique et classifiante et, par conséquent, nous avons affaire à des composées collocationnels.

\section{Contre vent et marée}

Comme nous l'avons vu, la relation entretenue entre le nom tête et l'adjectif classifieur (base et collocatif des composés collocationnels) pourrait être assimilée au binôme genre-espèce ( $c f$. nomenclature linnéenne) puisque :

Le nom tête nomme les propriétés générales communes à un ensemble d'individus, l'adjectif classifieur ajoute les propriétés spécifiques. [...] L'ensemble formé par le nom et l'adjectif classifieur ajoute des caractéristiques (celle de l'espèce) aux caractéristiques apportées par le nom seul (le genre), l'espèce est bien ici ce qui est rangé sous le nom (Girardin, 2005 : 68).

Si nous analysons la série de composés collocationnels marée noire, marée verte, marée rouge, nous pourrions constater que, l’AdjChr, outre le sème chromatique, ajoute des particularités spécifiques qui définissent chacune des espèces incluses dans le « genre » marée.

Tableau 4. Signifiés des UP ayant la structure marée + AdjChr

\begin{tabular}{ll}
\hline Marée noire & Écoulement en zone côtière d'une vaste nappe d'hydrocarbures. \\
Marée verte & $\begin{array}{l}\text { Important dépôt d'algues vertes nitrophiles sur les plages } \\
\text { Marée rouge }\end{array}$ \\
\hline
\end{tabular}

Or, force est de constater qu'a priori le nom tête marée n’apporte aucune caractéristique définissant un genre, d'autant plus que marée, en tant que 'nappe formée sur les eaux / plages', n'existe pas à l'état isolé11. À notre avis, il est fort possible que l'expression marée noire, inventée à l'occasion naufrage du Torrey Canyon ${ }^{12}$, soit incorporée tout d'abord comme un composé locutionnel, à savoir avec un sens unitaire. Néanmoins, vu l'existence de phénomènes qui, d'un point de vue empirique ressemblaient aux nappes d'hydrocarbure, par analogie, on a anéanti l'unité sémantique de l'UP et on a fini par associer l'AdjChr métonymiquement au sens 'provoqué par l'effet des hydrocarbures', la notion de 'nappe aquatique' restant attribué à marée. Aussi, les nouveaux termes marée rouge et marée verte ont ouvert le paradigme des marées 'nappes / couches aquatiques' en faisant de ces termes des noms composés collocationnels.

Cela dit, il nous semble plus logique d’affirmer que la classification opérée par les adjectifs classifieurs ne s'établirait pas

\footnotetext{
${ }^{11}$ En anglais, par exemple, alors qu'une marée noire est un oil spill (= renversement/ flaque de fuel), les marées rouges ou vertes sont des red tides / green tides (= marée rouge, marée verte ).

${ }^{12}$ https://fr.wikipedia.org/wiki/Torrey_Canyon [consulté le 15 avril 2016]. Le terme marée noire est déjà attesté en 1891 mais son signifié était ‘pas de lune’.
} 
sur l'axe vertical espèce-genre (une marée noire n'est pas un type de marée), mais plutôt sur l'axe horizontal organisant les co-hyponymes (la marée noire est semblable à la marée verte, mais avec des particularités). Cela nous évite de faire de faux amalgames entre termes dont les noms tête signifient des choses différentes ${ }^{13}$. Ainsi, par exemple, malgré le fait de partager une structure identique, nous devons faire une distinction entre la série marée ( (nappe aquatique'), e. g. marée verte, marée noire, marée rouge et la série néologique créée à l’occasion des mouvements revendicatifs espagnols où le mot marée 2 prend le sens de 'flot irrésistible que forme une masse de personnes qui se déplacent' (TLFi) :

\begin{abstract}
À Madrid, cinq cortèges conflueront aux portes des Cortes : la marée verte pour la défense de l'éducation universelle et gratuite, la marée blanche contre la privatisation du système de santé, la marée violette pour le respect des droits des femmes, la marée bleue contre la privatisation de la gestion de l'eau de la capitale, la marée noire composée des fonctionnaires, boucs-émissaires des politiques d'austérité. ${ }^{14}$
\end{abstract}

Bien que l'AdjChr qui accompagne la lexie marée dans cette deuxième série ait eu une justification métonymique à l'origine (la couleur des t-shirts des manifestants), de nos jours, la couleur n'est qu'une sorte de vestige d'une conventionnalisation idiosyncrasique. De fait, ces noms composés collocationnels sont devenus des appellations utilisées pour parler de l'ensemble d'actions organisées au sein d'un collectif travaillant en faveur des objectifs incarnés par les différentes couleurs. Cela implique la reconnaissance d'une nouvelle resémantisation du terme marée qui passe d’un 'cortège’ à un 'ensemble d'actions de mobilisation en faveur de certains objectifs' .

\title{
Conclusion
}

Nous avons pu constater que l'eau est un élément « vital » comme générateur de nouvelles UP. Le registre lexicographique de cette diversité, parfois héritier de certaines traditions assumées de manière dogmatique, peut pourtant s’avérer peu nette, voire trouble. Nous avons voulu mettre en évidence la nécessité d’appliquer un filtre un filtrage permettant de distinguer, malgré la perméabilité inhérente à ces unités, les sous-classes phraséologiques, étant donné que, sans doute, il devrait entraîner des répercussions au niveau de l’organisation de la microstructure des dictionnaires.

Tableau 5. Cadre récapitulatif

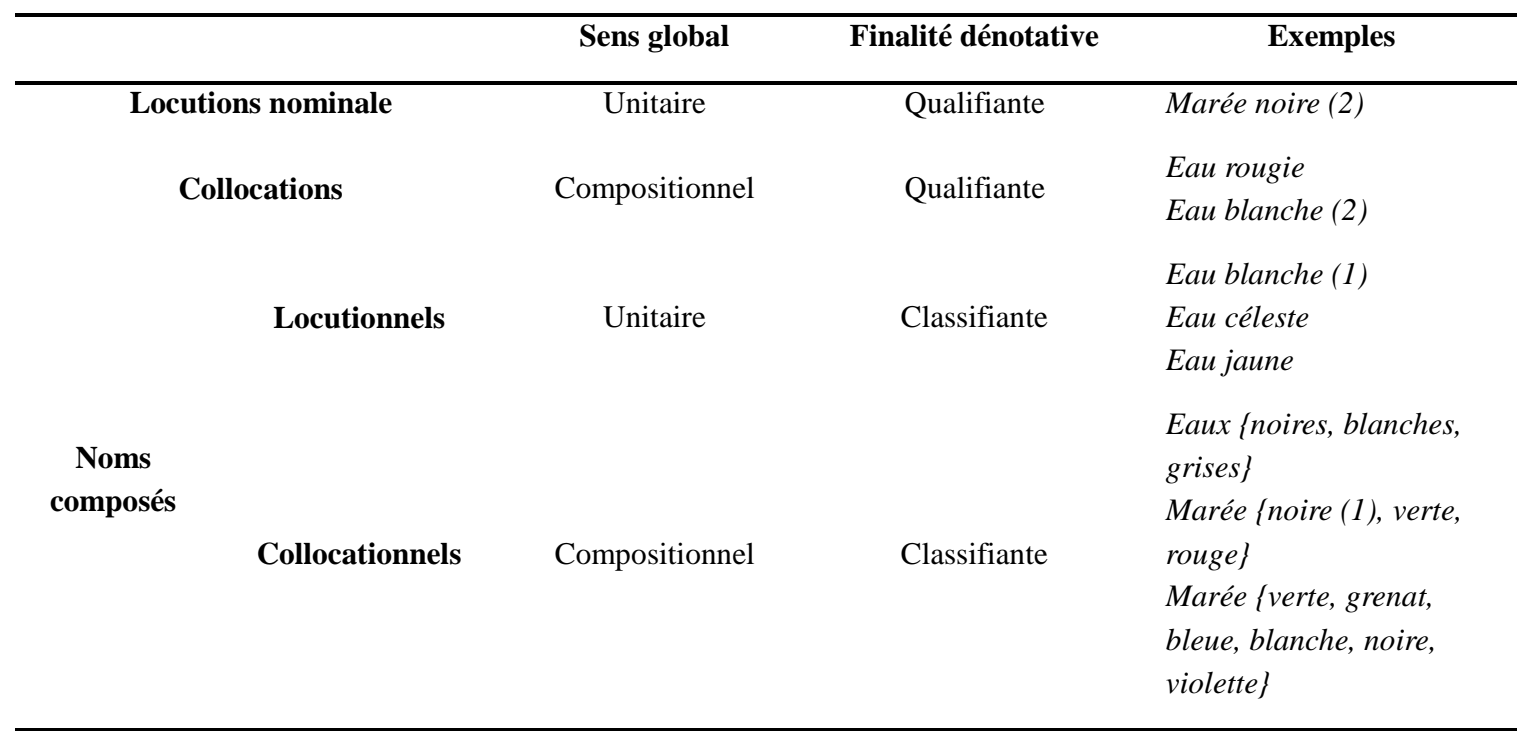

\footnotetext{
${ }^{13}$ Cf. en espagnol oso \{negro, pardo, blanco, polar, grizzli\} vs oso hormiguero.

${ }^{14}$ Il faudrait ajouter également la marée grenat des jeunes « exilés ». Source : http://www.humanite.fr/espagne-maree-citoyenne-contre-la-crise [consulté le 15 avril 2016].
} 
Un recensement exhaustif des sens des termes composant les UP, reflétant leur richesse polysémique, permettra d’organiser de manière efficace ce fourre-tout qui regroupe les « combinaisons fréquentes ».

\section{Références bibliographiques}

Anscombre, Jean-Claude (2011). « Figement, idiomaticité et matrices lexicales » dans Anscombre, Jean-Claude et Mejri, Salah (éds.) Le figement linguistique : la parole entravée. Paris : Honoré Champion.

Dobrovol'skiJ, Dimitrij et PirRainen, Elisabeth (2005). Figurative Language : CrossCultural and Cross-Linguistic Perspectives. Amsterdam : Elsevier.

EicheNlaub, Jean-Luc (2011). « Bleu, verte, rouge. Note sur les eaux d'Alsace en bouteille (vers 1990-2010) » Revue d’Alsace, $\mathrm{n}^{\circ} 137$ : Boissons en Alsace de l'Antiquité à nos jours, p. 285-290.

GIRARDiN, Charlotte (2005). « Les classifieurs : une sous-classe d’adjectifs non prédicatifs ». Cahiers de Lexicologie n 86, p. 59-70.

IÑEsta Mena, Eva María et Pamies Bertrán, Antonio (2002). Fraseología y metáfora : aspectos tipológicos y cognitivos. Granada : Granada Lingvistica.

LE Fur, Dominique et Freund, YaËL (éds.) (2007). Dictionnaire des combinaisons de mots : les synonymes en contexte, Collection Les Usuels, Paris : Dictionnaires Le Robert.

LEgAlLois, Dominique et TUTin, Agnès (éds.) (2013). Vers une extension du domaine de la phraséologie. Langages I/2013 (nº 189).

MEL’CuK, Igor (2013). « Tout ce que nous voulions savoir sur les phrasèmes, mais...». Cahiers de Lexicologie n 102, p. 129-149.

Pastoureau, Michel (1992). Dictionnaire des couleurs de notre temps. Paris : Bonneton.

Timofeeva, Larissa (2012). El significado fraseológico. En torno a un modelo explicativo y aplicado. Madrid : Liceus.

Tutin, Agnès (2013). « Les collocations lexicales : une relation essentiellement binaire définie par la relation prédicat-argument » dans Legallois et Dominique, Tutin, Agnès (éds.) Vers une extension du domaine de la phraséologie. Langages I/2013 (nº 189), p. 47-63.

WHITTAKeR, Sunniva (2002). La notion de gradation : Applications aux adjectifs. Bern, Berlin, Bruxelles : Peter Lang. 